Medeiros, MAC, Simões, MM, Santos, B, Marques, FMC, Leite, MFMS, Oliveira, HMBF, Oliveira, VF, Cunha, SMD, Maia, GLA, Sousa, AP \& Oliveira Filho, AAO. (2020). Evaluation of the photoprotective activity of the ethanolic extract of Rhaphiodon echinus Schauer. Research, Society and Development, 9(7): 1-10, e585974410.

\title{
Avaliação da atividade fotoprotetora do extrato etanólico de Rhaphiodon echinus
}

\section{Schauer}

Evaluation of the photoprotective activity of the ethanolic extract of Rhaphiodon echinus

Schauer

Evaluación de la actividad fotoprotectora del extracto etanólico de Rhaphiodon echinus Schauer

Recebido: 11/05/2020 | Revisado: 17/05/2020 | Aceito: 19/05/2020 | Publicado: 30/05/2020

Maurício André Campos de Medeiros

ORCID: https://orcid.org/0000-0003-0276-527X

Universidade Federal de Campina Grande, Brasil

E-mail: mauricioandre64@gmail.com

Mylena Medeiros Simões

ORCID: https://orcid.org/0000-0002-0654-4733

Universidade Federal de Campina Grande, Brasil

E-mail: mylenamedeirossimoes@gmail.com

Bernadete Santos

ORCID: https://orcid.org/0000-0003-4234-4752

Universidade Federal de Campina Grande, Brasil

E-mail: bernadetes672@gmail.com

Fernanda Matias Cariri Marques

ORCID: https://orcid.org/0000-0001-5571-1596

Universidade Federal de Campina Grande, Brasil

E-mail: fernandacariri20@gmail.com

Maria Francyherla Miguel da Silva Leite

ORCID: https://orcid.org/0000-0002-7968-7751

Universidade Federal de Campina Grande, Brasil

E-mail: francyherllaleiite@gmail.com 


\section{Heloisa Mara Batista Fernandes de Oliveira}

ORCID: https://orcid.org/0000-0001-8237-9920

Hospital Universitário Ana Bezerra, Brasil

E-mail: Heloisambf@gmail.com

Vinícius Filgueiras de Oliveira

ORCID: https://orcid.org/0000-0002-2584-3624

Universidade Federal da Paraíba, Brasil

E-mail: filgueirasv@gmail.com

Saraghina Maria Donato da Cunha

ORCID: https://orcid.org/0000-0002-3015-6175

Universidade Federal da Paraíba, Brasil

E- mail: saracunha20@ hotmail.com

Gabriela Lemos de Azevedo Maia

ORCID: https://orcid.org/0000-0002-6878-4644 Universidade Federal do Vale do São Francisco, Brasil

E- mail: gabriela.lam@gmail.com

Aleson Pereira de Sousa

ORCID: https://orcid.org/0000-0002-3430-477X

Universidade Federal da Paraíba, Brasil

E-mail: aleson_155@hotmail.com

Abrahão Alves de Oliveira Filho

ORCID: https://orcid.org/0000-0002-7466-9933

Universidade Federal de Campina Grande, Brasil

E-mail: abrahao.farm@gmail.com

\section{Resumo}

O objetivo do presente estudo foi avaliar, in vitro, a atividade fotoprotetora do extrato etanólico de Rhaphiodon (R.) echinus. O experimento foi realizado com diferentes concentrações do produto natural $(50,100,500$ e $1000 \mu \mathrm{g} / \mathrm{mL})$. Através de varreduras com o auxílio de um espectrofotrometro, nas faixas (290 a $320 \mathrm{~nm}$ ) com intervalos de $5 \mathrm{~nm}$. Os dados foram submetidos a equação de Mansur e colaboradores (1986). De acordo com os resultados obtidos, apenas as duas maiores concentrações $(500 \mu \mathrm{g} / \mathrm{mL}$ e $1000 \mu \mathrm{g} / \mathrm{mL})$ apresentaram valores de fotoproteção consideráveis e acima do fator mínimo de proteção de 6 (FPS), sendo 23,65 e 25,00 respectivamente. Com isso, a atividade fotoprotetora do extrato 
etanólico, in vitro, de R. echinus demonstra uma importante característica bioativa. Ademais, o resultado ainda sugere uma possível utilização dessa planta em formulações fitocosméticas, pois o uso de plantas como fotoprotetoras é uma das alternativas acessíveis.

Palavras - Chave: Fotoproteção; Fitoterapia; Produto natural.

\begin{abstract}
The aim of the present study was to evaluate, in vitro, the photoprotective activity of the ethanolic extract of Rhaphiodon (R.) echinus. The experiment was carried out with different concentrations of the natural product $(50,100,500$ and $1000 \mu \mathrm{g} / \mathrm{mL})$. Through scans with the aid of an spectrophotometer, in the ranges $(290$ to $320 \mathrm{~nm})$ with $5 \mathrm{~nm}$ intervals. The data were submitted to Mansur et al. (1986) equation. According to the results obtained, only the two highest concentrations $(500 \mu \mathrm{g} / \mathrm{mL}$ and $1000 \mu \mathrm{g} / \mathrm{mL})$ showed considerable photoprotection values and above the minimum protection factor of 6 (SPF), being 23.65 and 25.00 respectively. Thus, the photoprotective activity of the ethanol extract, in vitro, of $R$. echinus demonstrates an important bioactive characteristic. Furthermore, the result still suggests a possible use of this plant in phytocosmetic formulations, since the use of plants as photoprotectors is one of the accessible alternatives.
\end{abstract}

Key words: Photoprotection; Phytotherapy; Natural product.

\title{
Resumen
}

El objetivo del presente estudio fue evaluar, in vitro, la actividad fotoprotectora del extracto etanólico de Rhaphiodon (R.) echinus. El experimento se realizó con diferentes concentraciones del producto natural $(50,100,500$ y $1000 \mu \mathrm{g} / \mathrm{ml})$. A través de escaneos con la ayuda de un espectrofotómetro, en los rangos (290 a $320 \mathrm{~nm}$ ) con intervalos de $5 \mathrm{~nm}$. Los datos se presentaron a la ecuación Mansur et al. (1986). Según los resultados obtenidos, solo las dos concentraciones más altas $(500 \mu \mathrm{g} / \mathrm{mL}$ y $1000 \mu \mathrm{g} / \mathrm{mL})$ mostraron valores de fotoprotección considerables y superiores al factor de protección mínimo de 6 (SPF), siendo 23.65 y 25.00 respectivamente. Por lo tanto, la actividad fotoprotectora del extracto etanólico, in vitro, de $R$. echinus demuestra una importante característica bioactiva. Además, el resultado aún sugiere un posible uso de esta planta en formulaciones fitocosméticas, ya que el uso de plantas como fotoprotectores es una de las alternativas accesibles.

Palabras clave: Fotoprotección; Fitoterapia; Producto natural. 


\section{Introdução}

A camada de ozônio está intimamente ligada a absorção da radiação ultravioleta do tipo B (UV-B), sendo fundamental, uma parte dessa radiação que alcança o plano terrestre, todavia, o que excede desses raios pode favorecer o surgimento de neoplasias na pele, chamando a atenção de especialistas na área da saúde (Tomasoni, 2010).

Segundo Gontijo, Pugliesi e Araújo (2009) a utilização de filtros solares precisa, habitualmente, fazer parte da prevenção contra raios solares na pele, começando principalmente na infância. Entretanto, manter uma utilização regular de fotoprotetores no Brasil torna-se economicamente difícil para uma grande parte da população, pois o custo desses produtos possui um valor pouco acessível ao consumidor (De Souza \& Brandão, 2019).

A utilização desses produtos não pode ser descartada, pois os filtros solares atuam inibindo os efeitos danosos do excesso da radiação que incide na pele, evitando o envelhecimento da pele e neoplasias nesse tecido que reveste o corpo (Tofetti \& Oliveira, 2006). Todavia, é fundamental a informação de possíveis efeitos colaterais, bem como a possibilidade de causar irritações na pele (Santos, Sobrinho, \& Oliveira, 2018).

Dessa forma, a quantidade de estudos tem crescido com o passar do tempo, objetivando estruturar substâncias que tenham resultados positivos contra os raios solares ultravioletas que atingem nossa atmosfera (Balogh et al., 2011). Neste contexto, o desenvolvimento de novos fármacos, predominantemente associada ao estudo de produtos naturais, está favorecendo descobertas e avanços na medicina e demais áreas cientificas (Berlinck, Borges, Scotti, \& Vieira, 2017).

A utilização de extratos provenientes de plantas medicinais, no desenvolvimento de produtos fotoprotetores, pode agregar novas substâncias que apresentem atividade na fotoproteção, sustentabilidade ao fazer uso da biodiversidade do país e um preço mais acessível à população (Polinini, Raposo, \& Brandão, 2011).

Muitos trabalhos têm sido feitos abrangendo compostos orgânicos, produto do metabolismo secundário de inúmeras plantas, destacando-se compostos fenólicos como flavonoides, taninos e os terpenos (Cunha, Moura, Barbosa, \& Santos, 2016). Na fotoproteção, os flavonoides são majoritariamente o grupo de metabólitos secundários que possuem efeito mais ressaltante bem como diminuem a produção de radicais livres (Orlanda \& Vale, 2015). 
No Brasil, há uma enorme diversidade de plantas, dentre as quais, encontram-se as plantas da família Lamiaceae que tem distribuição em muitos domínios fitogeográficos do país (Flora do Brasil, 2020). A espécie Rhaphiodon echinus, conhecida popularmente como "betônica", que pertence a esse grupo taxonômico, tem propriedades medicinais e possui em sua composição fitoquímica os flavonoides como um dos produtos do seu metabolismo secundário (PIO et al., 2019).

Com base em todas as informações relacionadas ao uso de produtos naturais e a fotoproteção, esse trabalho tem como objetivo, avaliar o efeito, in vitro, fotoprotetor, do extrato etanólico de Rhaphiodon echinus.

\section{Metodologia}

O presente estudo trata-se de uma pesquisa aplicada, experimental e quantitativa. Günther (2006) definiu como pesquisa quantitativa utiliza dados coletados por meio do uso de medições de valores, a mensuração realizada através de números com suas respectivas unidades. Logo, há necessidade da aplicação desse método para verificação dos resultados obtidos a partir dos objetivos propostos no estudo.

\section{Extrato Vegetal}

Para o desenvolvimento do estudo in vitro, utilizou-se o extrato etanólico que foi cedido pela equipe da Prof ${ }^{\mathrm{a}}$. Dr ${ }^{\mathrm{a}}$. Gabriela Lemos de Azevedo Maia da Universidade Federal do Vale do São Francisco.

\section{Avaliação do Fator de Proteção Solar (FPS)}

A análise espectrofotométrica de absorção utilizando o extrato etanólico de Rhaphiodon echinus realizou-se no espectro da radiação ultravioleta como proposto por Mansur e colaboradores (1986), com isso, efetuou-se varreduras de 290 a 320 nm, atribuindose intervalos de $5 \mathrm{~nm}$ com duração de 5 minutos. Ao final de cada intervalo foi realizado a mensuração das absorbâncias.

$\mathrm{Na}$ obtenção da leitura, utilizou-se o espectrofotômetro digital (Biospectro®) com cubeta de quartzo de $1 \mathrm{~cm}$. Finalizada a mensuração das absorbâncias, os dados foram submetidos a equação de Mansur e colaboradores (1986) para aferir o Fator de Proteção Solar (FPS) in vitro. Esse método coloca em lista o efeito eritomatogênico e a intensidade da 
radiação (EE x I) que foram medidos por Sayre e colaboradores (1979), esses são demonstrados no Quadro 1, abaixo:

Quadro 1 - Relação efeito eritemogênico (EE) versus intensidade da radiação (I) conforme o comprimento de onda $(\chi)$.

\begin{tabular}{|c|c|}
\hline$\lambda / \mathrm{nm}$ & EE x I \\
\hline 290 & 0,0150 \\
\hline 295 & 0,0817 \\
\hline 300 & 0,2874 \\
\hline 305 & 0,3278 \\
\hline 310 & 0,1864 \\
\hline 315 & 0,0839 \\
\hline 320 & 0,0180 \\
\hline
\end{tabular}

Fonte: Sayre, Agin, LeVee, \& Marlowe (1979).

Sendo que a fórmula de Mansur e colaboradores (1986) é também composta pela leitura espectrofotométrica da absorbância da solução e fator de correção (=10). Essa equação pode ser observada, a seguir:

FPS espectrofotométrico $=$ FC. $\Sigma$ EE $(\lambda)$. I $(\lambda)$. Abs $(\lambda)$

Na qual: FPS = fator de proteção solar; FC = fator de correção, calculado de acordo com dois filtros solares de FPS conhecidos e testados em seres humanos de tal forma que um creme contendo $8 \%$ de homossalato resultasse no FPS 4; $\mathrm{EE}(\lambda)=$ efeito eritemogênico da radiação de comprimento de onda; $I(\lambda)=$ a intensidade da luz solar no comprimento de onda e $\operatorname{Abs}(\lambda)=$ a absorbância da formulação no comprimento de onda.

\section{Resultados e Discussão}

Os resultados da análise in vitro do Fator de Proteção Solar (FPS) presente em extrato etanólico de Rhaphiodon echinus pode ser visto na Tabela 1 . As concentrações de $(500$ e 1000 $\mu \mathrm{g} / \mathrm{mL}$ ) foram as que apresentaram melhor efeito fotoprotetor para o produto natural testado. 
(CC BY 4.0) | ISSN 2525-3409 | DOI: http://dx.doi.org/10.33448/rsd-v9i7.4410

Tabela 1 - FPS do extrato etanólico de Rhaphiodon echinus em diferentes concentrações.

\begin{tabular}{cllll}
\hline TOTAL & $50 \mu \mathrm{g} / \mathrm{mL}$ & $100 \mu \mathrm{g} / \mathrm{mL}$ & $500 \mu \mathrm{g} / \mathrm{mL}$ & $1000 \mu \mathrm{g} / \mathrm{mL}$ \\
\hline FPS & $\mathbf{3 , 4 0}$ & $\mathbf{5 , 1 5}$ & $\mathbf{2 3 , 6 5}$ & $\mathbf{2 5 , 0 0}$
\end{tabular}

Fonte: Os Autores, 2020.

Conforme a RDC $\mathrm{N}^{\mathrm{o}} 30$, de $1^{\mathrm{o}}$ de junho de 2012, da Agência Nacional de Vigilância Sanitária (ANVISA), ao ser aprovado o regulamento técnico Mercosul sobre protetores solares em cosméticos e dá outras providências, atribui-se uma regulamentação técnica aplicada aos produtos e cosméticos destinados a proteção solar da pele, que, apresenta o fator mínimo de proteção solar igual a 6 (seis) (FPS).

Com base na análise dos dados apresentados, observa-se que apenas as duas maiores concentrações $(500 \mu \mathrm{g} / \mathrm{mL}$ e $1000 \mu \mathrm{g} / \mathrm{mL})$ apresentaram valor de fator de fotopoteção acima de 6 FPS, com os valores de 23,65 e 25,00 respectivamente.

O extrato etanólico de Rhaphiodon echinus apresentou outras atividades biológicas, como a atividade antifúngica no combate a cepas de Candida tropicalis, mostrando-se eficiente na ação fungicida (Ferreira et al., 2019). Demostrando assim o potencial biológico desse produto natural.

Um estudo, in vitro, sobre fotoproteção, utilizando o extrato etanólico da espécie Ocimum gratissimun L., conhecida popularmente como Alfavaca, apresentou propriedades fotoprotera e antioxidante (Vieira \& Orlanda, 2018). Esta espécie pertence ao grupo taxonômico “família” Lamiaceae (Flora do Brasil, 2020), reforçando assim este estudo com Rhaphiodon echinus.

\section{Considerações Finais}

A partir dos dados obtidos nessa pesquisa, pode-se observar que o extrato etanólico da espécie Rhaphiodon echinus, apresenta uma atividade promissora como fotoprotetor. No entanto, mais estudos, in vivo, precisam ser feitos para confirmar esse potencial biológico e identificar o mecanismo de ação desta atividade. 


\section{Referências}

Balogh, T.S., Velasco, M.V.R., Pedriali, C.A., Kaneko, T.M. \& Baby, A.R. (2011) Proteção à radiação ultravioleta: recursos disponíveis na atualidade em fotoproteção. Anais brasileiros de Dermatologia, 86(4), 732-742.

Berlinck, R.G.S., Borges, W.S., Scotti, M.T. \& Vieira, P.C. (2017) A química de produtos naturais do Brasil do século XXI. Química Nova, 40(6), 706-710.

Brasil. (2012) Agência Nacional de Vigilância Sanitária. Resolução RDC No 30, de $1^{\text {o }}$ de junho de 2012. Aprova Regulamento técnico do MERCOSUL sobre protetores solares em cosméticos. Diário Oficial da União. Brasília, DF.

Cunha, A.L., Moura, K.S., Barbosa, J.C. \& Santos, A.F. (2016) Os metabólitos secundários e sua importância para o organismo. Diversitas Journal, 1(2),175-181.

Ferreira, J.L.S. et al. (2019) Comparação da atividade antifúngica do extrato aquoso e do extrato etanólico de Rhaphiodon echinus (Lamiaceae) contra cepas Candida tropicalis. Revista da Universidade Vale do Rio Verde, 17(1).

Gontijo, G.T., Pugliesi, M.C.C. \& Araújo, F.M. (2009) Fotoproteção. Surgical \& Cosmetic Dermatology, 1(4) 186-192.

Günther, H. (2006). Pesquisa qualitativa versus pesquisa quantitativa: esta é uma questão? Psicologia: Teoria e Pesquisa, 22 (2), 201-209.

Pastore, J.F.B. \& Mota, M.C.A. Rhaphiodon in Flora do Brasil 2020 em construção. Jardim Botânico do Rio de Janeiro. Disponível em:

〈http://floradobrasil.jbrj.gov.br/reflora/floradobrasil/FB8295>. Acesso em: 04 mai. 2020

Mansur, J.S., Breder, M.N.R., Mansur, M.C.A. \& Azulay, R.D. (1986) Determinação do fator de proteção solar por espectrofotometria. An. Bras. Dermatol, 61(3), 121-124. 
Ocimum in Flora do Brasil 2020 em construção. Jardim Botânico do Rio de Janeiro.

Disponível em: 〈http://floradobrasil.jbri.gov.br/reflora/floradobrasil/FB23332〉. Acesso em: 08 Abr. 2020.

Orlanda, J.F.F. \& Vale, V.V. (2015) Análise fitoquímica e atividade fotoprotetora de extrato etanólico de Euphorbia tirucalli Linneau (Euphorbiaceae). Revista Brasileira de Plantas Medicinais, 17(4), 730-736.

Pio, I.D.S.L. et al. (2019) Traditional knowledge and uses of medicinal plants by the inhabitants of the islands of the São Francisco river, Brazil and preliminary analysis of Rhaphiodon echinus (Lamiaceae). Braz. J. Biol. 79(1), 87-99. Disponível em:

$<$ http://www.scielo.br/scielo.php?script=sci_arttext\&pid=S151969842019000100087\&lng=en\&nrm=iso>. Acesso em: 23 Jan. 2020.

Polonini, H.C., Raposo, N.R.B. \& Brandão, M.A.F. (2011) Fotoprotetores naturais como instrumento de ação primária na prevenção do câncer de pele. Revista APS, 14(2), 216-223.

Santos, S.O., Sobrinho, R.R. \& De Oliveira, T.A. (2018) Importância do uso de protetor solar na prevenção do câncer de pele e análise das informações desses produtos destinados a seus usuários. Journal of Health \& Biological Sciences, 6(3), 279-285.

Sayre, R.M., Agin, P.P., LeVee, G.J. \& Marlowe, E. (1979) A Comparison of in vivo and in vitro testing of sunscreening formulas. Photochemistry and Photobiology, 29(3), 559-566.

Tofetti, M.H.F.C. \& De Oliveira, V.R. (2006) A importância do uso do filtro solar na prevenção do fotoenvelhecimento e do câncer de pele. INVESTIGAÇÃO, 6(1).

Tomasoni, M. A. (2011) Mudanças globais: a problemática do ozônio e algumas de suas implicações. GeoTextos, 7(2).

Vieira, C.B.S. \& Orlanda, J.F.F. (2018) Atividade antioxidante e fotoprotetora do extrato etanólico de Ocimum gratissimum L. (alfavaca, Lamiaceae). Revista Cubana de Plantas Medicinales, [S.1.], 23(3). Disponível em: 
<http://revplantasmedicinales.sld.cu/index.php/pla/article/view/626/319>. Acesso em: 08 abr. 2020.

\section{Porcentagem de contribuição de cada autor no manuscrito}

Maurício André Campos de Medeiros - 10\%

Mylena Medeiros Simões - 9\%

Bernadete Santos - 9\%

Fernanda Matias Cariri Marques - 9\%

Maria Francyherla Miguel da Silva Leite - 9\%

Heloisa Mara Batista Fernandes de Oliveira - 9\%

Vinícius Filgueiras de Oliveira - 9\%

Saraghina Maria Donato da Cunha - 9\%

Gabriela Lemos de Azevedo Maia - 9\%

Aleson Pereira de Sousa - 9\%

Abrahão Alves de Oliveira Filho - 9\% 